\title{
Deafness and Its Burdens - Prevention and Control
}

\author{
Dr. Shuaib Kayode Aremu, MBBS, Cert LMIH, FWACS \\ ENT Department, Federal teaching Hospital Ido-Ekiti/Afe Babalola University, Ado- Ekiti \\ "Corresponding Author: Dr Shuaib Kayode Aremu; shuaib.aremu@gmail.com
}

Received 08 March 2019;

Accepted 04 April 2019;

Published 12 April 2019

\begin{abstract}
There is high burden of deafness globally is largely preventable and may be avoidable. Over 5\% of the population of the world (466 million people) are living with disabling hearing loss (432 million adults and 34 million children). In 2002 WHO noticed that hearing loss has become a burden globally and major attentions were being paid on children and youth with adult being neglected.

Hearing loss is the second most common cause of years lived with disability .Hearing impairment occurs when there is reduction in hearing acuity. Hearing impairment is a neglected chronic otological disorder with varying aetiology. Permanent childhood hearing loss is a significant health condition and its detection through screening with oto-acoustic emissions and/or auditory brainstem response is feasible and made early intervention possible and rewarding.

Nationwide disability surveys estimated that hearing loss is the second most common cause of disability. This has posed a greater challenge to the practice of otolaryngology worldwide. A lack of skilled manpower, human resources and diagnostic facilities make this problem a huge challenge.
\end{abstract}

Keywords: Hearing, Hearing loss, Deafness, Hearing disability, HearWHO

\section{Introduction}

Hearing loss/Deafness is the most frequent sensory loss in human population occurring in over 250 million worldwide.

Over $5 \%$ of the population of the world (466 million people) are living with disabling hearing loss (432 million adults and 34 million children). By 2050 an estimate of over 900 million people (or one in every ten people) will have disabling hearing loss. ${ }^{[1]}$

Hearing loss is associated with a lot of burdens and this include ${ }^{[2]}$

1. Delayed language acquisition

2. Speech interpretation disorder

3. Economic and social problem

4. Social stigmatization

In 2002 WHO noticed that hearing loss has become a burden globally and major attentions were being paid on children and youth with adult being neglected. ${ }^{[1]}$

For these reasons, this year's theme is focused on the elderly.

\section{Definitions}

Hearing loss: refer to diminish sensitivity to sound within speech frequency and this may be mild, moderate, severe or profound in nature. $^{[3]}$
Deafness: there is hearing impairment of severe or profound nature with limitation in the day to day activities of an individual (quality of life is being affected). ${ }^{[3]}$

\section{Physiology of Hearing}

The Human ear has 3 parts: the outer ear, the middle ear, and the inner ear.

The outer ear consists of the pinna which collects the sound waves and sends it through the external auditory canal at the end of which there is a tympanic membrane (ear drum) which amplifies the sound and transmit it to the 3 ossicles in the middle ear. The sound is subsequently passed via the oval window into the cochlea where the sound waves are converted to an electrical signal that is conducted by the auditory nerve to the auditory center in the brain where the sound is interpreted and given meanings.

Problems along these pathways will result in hearing loss.

\section{Types of Hearing Loss}

A. Conductive - Due to Problems along the conductive pathway

B. Sensory neural -Due to problems along the neural pathway

C. Mixed - Combination of A and B 


\section{Specific Causes of Hearing Loss}

1. Age. There is an entity called presbyacusis which agerelated hearing loss. Age is associated with degenerative changes in different part of the body and ear is not an exception. The problem in middle and progress and initially the quality of life is not affected but it is so with time.

2. Noise. Noise-induced hearing loss accounts for about $50 \%$.

It occurs in $5 \%$ population. ${ }^{[4]}$ Populations who reside close to the airports or freeways are exposed to levels of noise in the range of 65 to $75 \mathrm{~dB}(\mathrm{~A})$., Prolonged outdoor lifestyle and exposures over time can degrade hearing. Noise standards have been set by the U.S.EPA and other states to protect people from these adverse health risks. Exposure to Sound level of $70 \mathrm{~dB}(\mathrm{~A})$ have been documented by EPA for 24-hour as the level necessary to protect the public from hearing loss and other disruptive effects from noise, such as disturbance of sleep, stressrelated problems, learning detriment, etc. (EPA, 1974). ${ }^{[5]}$ Risk factors include nearness to airport and high way. Working is steel rolling companies. Loud generator noise and earphones usage

3. Congenital/hereditary disorders like microtia, Eustachian tube stenosis, and absence of cochlear.

4. Diseases like measles, mumps, meningitis, HIV/AIDS, chlamydia. Intra-uterine infections. The fetal alcohol syndrome is associated with hearing loss in $64 \%$ of cases

5. Drug overdose. Drugs such as antimalarial (chloroquine). antibiotics(gentamicin), NSAID, anti-neoplastic and diuretics

6. Heavy metals. Eg Mercury and Lead. Also chemicals like herbicides, pesticides etc.

7. Trauma such as Head injury, bomb blast, and barotrauma

8. Others- Wax Impaction, foreign body impaction etc.

\section{Management of Hearing Loss}

Careful evaluation by taking an adequate history and physical examination. otoscopy, tuning fork test and PTA to obtain the quantitative and qualitative analysis of hearing loss.

Electrocochleography, BERA etc can also be done.

\section{Treatment}

This largely depends on the causes.

Wax impaction-Syringing

CSOM-aural toileting and antibiotic dressing

Sensory neural hearing loss-Nicotinic acid and neurobion tablets

Hearing Aid is important and beneficial to amplify sound for the patient.

Cochlear implant for direct stimulation of the auditory nerve
Surgical options include Mastoidectomy, ossicular prosthesis etc.

\section{Rehabilitative Measures}

These include lips reading, sign language, telecommunication devises for the deaf, instant internet messengers, videophones, video relay messages. hearing dogs etc.

\section{Prevention}

Prevention is better than cure. The Measures are:

1. Screening in neonate at 5,10 15 and 25 years of age

2. Immunization-pregnant women; and neonatal and childhood immunization programme.

3. Avoid risk factors: Avoid loud noise, foreign body insertion into the ear, ear cleaning with cotton buds, slapping the ear and self-medication.

4. WHO has highmarked every $3^{\text {rd }}$ of March as the world hearing day which is been celebrated all over the world to improve the awareness on hearing loss and its prevention. A new 'HearWHO' app has been developed by WHO for individuals to screen their hearing. This is quite impressive but not a substitute to the standard ontological screening by the otologists/audiologists.

\section{Conclusion}

Hearing loss though sound scaring it should be noted that many of the causes are preventable and treatable and in cases when not treatable there are varieties of rehabilitative measures to improve the quality of life of the affected individual.

\section{References}

[1] WHO. Deafness and hearing loss.2018. https://www.who.int/news-room/factsheets/detail/deafness-and-hearing-loss

[2] Blazer DG, Domnitz S, Liverman CT. Hearing Health Care for Adults: Priorities for Improving Access and Affordability. National Academies Press (US); 2016 Sep 6.

[3] Dobie RA, Van Hemel S. Hearing Loss: Determining Eligibility for Social Security Benefits. National Academies Press (US); 2004.

[4] Trung NL, Louise VS, Jane L, Brian Westerberg. Current insights in noise-induced hearing loss: a literature review of the underlying mechanism, pathophysiology, asymmetry, and management options. J Otolaryngol Head Neck Surg. 2017; 46: 41.

[5] Monica SH, Tracy KS, Richard LN. Environmental Noise Pollution in the United States: Developing an Effective Public Health Response. Environ Health Perspect. 2014 Feb; 122(2): 115-119. 